\title{
Effect of inclination angle on the performance of a kind of vapor chamber
}

\author{
Jinlong Ma, Xing Fu, Run Hu and Xaobing Luo*
}

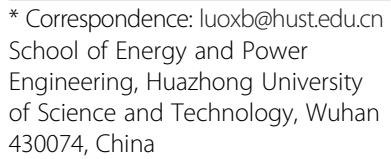

* Correspondence: luoxb@hust.edu.cn School of Energy and Power Engineering, Huazhong University of Science and Technology, Wuhan 430074, China

\begin{abstract}
Vapor chamber is an effective heat spreader for decreasing the hotspot phenomena in LED, and there are many parameters influencing its performance. In this paper, a vapor chamber was experimentally investigated. Both the thermal resistance and the temperature uniformity of the condensing surface under different inclination angles were tested. As the inclination angle changed from $0^{\circ}$ to $50^{\circ}$ with $10^{\circ}$ as an interval, the maximum relative change rate of thermal resistance and temperature uniformity were $9.1 \%$ and $4.5 \%$, respectively. And the relative standard deviations of them were $5.5 \%$ and $3.7 \%$.
\end{abstract}

\section{Background}

As electronic products become faster and incorporate more functions, they are simultaneously shrinking in size and weight. This results in a significant increase in heat generation. For such a case, when very small size heat sources exist, usually there was a large spreading thermal resistance from heat source to substrate or metal heat sink. It is necessary to find some substrates or heat sinks which can effectively decrease hotspot phenomena. The vapor chamber is such an effective solution to decrease the spreading thermal resistance because of its characteristics such as good temperature uniformity.

Many investigations were conducted to study the performance of the vapor chamber. Go [1] evaluated the thermal performance of a vapor chamber heat sink containing new microwick structures and found that it had a heat removal capacity of 80 (W/ $\mathrm{cm} 2)$ at the junction temperature of $85\left({ }^{\circ} \mathrm{C}\right)$ and an ambient temperature of $24\left({ }^{\circ} \mathrm{C}\right)$. Koito et al. [2] described the effect of heat source size on the thermal resistance of the vapor chamber in their experiments and simulations. They found although the thermal resistance is hardly affected by the heat generation rate and the heat flux of the heat source, it increases as the heat source become smaller. Wu et al. [3] made a fundamental thermal analysis of the vapor chamber, and put forward the factors that would impact the performance of heat sink. Zhang et al. [4] designed a novel grooved vapor chamber. The influence of heat flux, filling amount and gravity on the thermal performance was investigated by experiments. Huang et al. [5] experimentally investigated the thermal performance of the vapor chamber module applied to high power lightemitting diodes in natural convection. The results showed that the spreading resistance and the corresponding temperature difference of the flat-plate vapor chamber at $30 \mathrm{~W}$

(c) 2014 Ma et al.; licensee Springer; licensee Springer. This is an Open Access article distributed under the terms of the Creative Commons Attribution License (http://creativecommons.org/licenses/by/4.0), which permits unrestricted use, distribution, and reproduction in any medium, provided the original work is properly cited. 
were lower than those of the copper plate by $34 \%$ and $4\left({ }^{\circ} \mathrm{C}\right)$ respectively and the flat-plate vapor chamber could effectively lower the spreading resistance and diminish the hotspot effect. Wong et al. [6] experimentally investigated the performance of a novel vapor chamber with different working fluids of water, methanol and acetone. And they found water presents the smallest vapor chamber resistance while acetone has the largest.

The present authors have already done some researches on a vapor chamber which was applied to the thermal management of high power LED light source [7]. Experimental tests, numerical simulation were conducted and the results showed that the vapor chamber coupled fin heat sink could make the temperature of the fin base plate uniform and the thermal resistances of vapor chamber was $0.654(\mathrm{~K} / \mathrm{W})$ at the ambient temperature of $27\left({ }^{\circ} \mathrm{C}\right)$. However, the vapor chamber in that study was fabricated by conventional machining methods, thus the vapor chamber was large and heavy. Then we continued this part of work. The vapor chamber was fabricated by copper powder sintering and diffusion bonding processes. Its size sharply decreased. These samples will be used for the experimental investigation in this paper.

Based on the literature review on the existing vapor chamber work, it is found that most of the papers do not investigate the temperature uniformity under inclination condition, actually, this is very important in many application cases. For example, when we use vapor chamber to solve the hotspot phenomena and decrease the spreading thermal resistance in lighting emitting diodes packaging or other high heat flux chip packaging, the application situations often require the vapor chamber operation in inclination condition. In this case, if the temperature uniformity greatly changes or loses, thermal resistance is different with that in normal horizontal case, the cooling performance will sharply decrease, some of the chips will fail because of high temperature gradient.

In this paper, we will test the temperature characteristics of our small size vapor chamber under inclination conditions. The experimental setup was built, and the experimental results proved that our new version vapor chamber could achieve good thermal performance even under great inclination conditions.

\section{Methods}

\section{Vapor chamber and its principle}

Figure 1 shows the working mechanism of vapor chamber. A vapor chamber is a vacuum vessel with a wick structure distributing on the inside walls and the chamber is partly filled

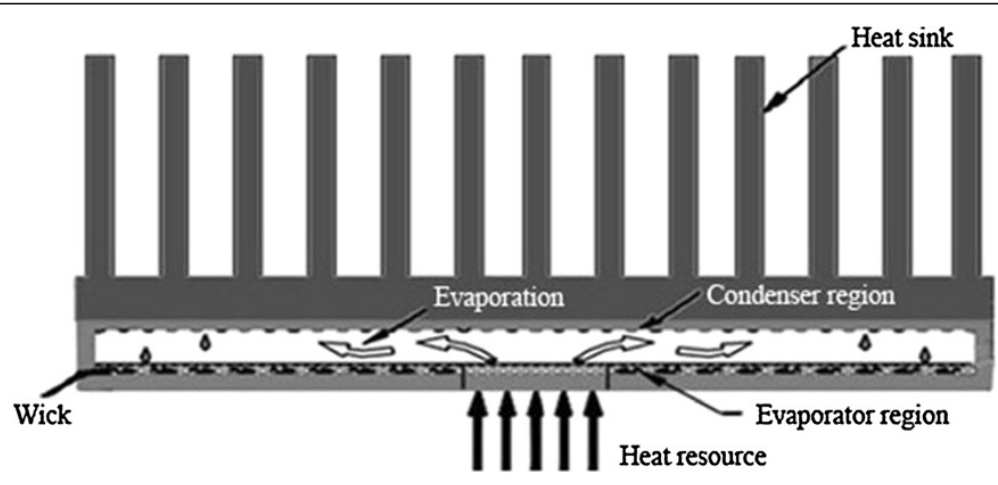

Figure 1 Operation schematic of vapor chamber. 
with working liquids. As heat is applied, the liquid at the evaporator region absorbs the heat from the heat resource and evaporates, then the resulting vapor flows to fill the empty part of the cavity. Whenever the vapor comes into contact with a cooler wall surface, it will condense and release the latent heat to the heat sink, finally the condensed liquids return to the evaporator region via the capillary force of the wick. The area of the heat sink is usually several fold or several tenfold of that of the heat source. The net effect of vapor chamber makes the heat transported from the evaporator to the condenser region and in this way the heat generated by heat resource could be diffused to the heat sink where the heat flux density decrease remarkably. Because of the flowing vapor inside the chamber, the temperature of the top surface of the chamber is uniform theoretically and the temperature of the heat sink can be uniform.

The vapor chamber designed and fabricated by our group mainly contained chamber body, wick sheet, wick columns and support column, as shown in Figure 2. The chamber was made of copper. The wick sheet was bonded on all six inner walls. There were four wick columns in the inner to increase the cycle efficiency of working liquid. Both the wick sheet and wick column were based on copper powder sintering, and they were made of small copper powders. Center support column was copper column, it was used for increasing the stiffness and decreasing the deformation. In order to ensure both the heat dissipation and the start performances of vapor chamber, the acetone was chosen as working liquid. Table 1 gives the size specifications of the vapor chamber.

\section{Experimental setup}

The experimental system contained five parts: heating part, thermal insulation part, vapor chamber, cooling part and data acquisition part. Its schematic diagram is shown in Figure 3. The heating part included aluminum bulk and copper rod. There were two heaters inserted in the aluminum bulk for heating supply. Cotton was wrapped around the aluminum bulk and copper rod for thermal insulation. The test vapor chamber was located on the copper rod. A fan was utilized to cool the condensing surface of the vapor chamber. In the system, the heat generated by the heater was conducted to the slender copper rod. Then the heat constrained in small area copper rod spreads to the bottom surface of the vapor chamber and finally to the top surface and the ambient.

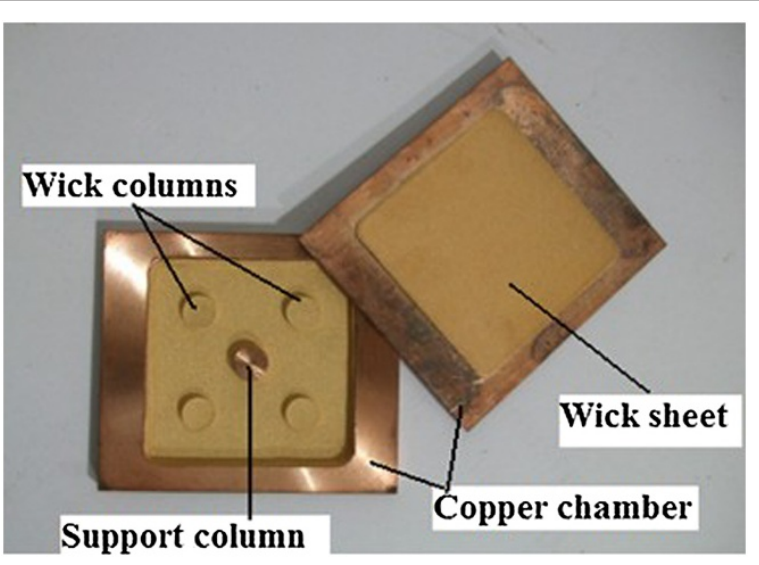

Figure 2 Picture of the vapor chamber. 
Table 1 Size specifications of the vapor chamber

\begin{tabular}{ll}
\hline Subject & Size \\
\hline Exterior bulk & $70 \times 80 \times 6 \mathrm{~mm}^{3}$ \\
Interior chamber & $60 \times 60 \times 4 \mathrm{~mm}^{3}$ \\
Top and bottom plate thickness & $1 \mathrm{~mm}$ \\
Wick sheet thickness & $0.5 \mathrm{~mm}$ \\
Wick (support) column radius & $5 \mathrm{~mm}$ \\
Wick (support) column height & $4.5 \mathrm{~mm}$ (based on bottom surface) \\
\hline
\end{tabular}

In this system, all the temperatures were measured by T-type thermocouples, and the data were transmitted to the computer through the digital data acquisition instrument. Figure 4 shows the thermocouple distribution points in copper rod and on vapor chamber surfaces. As the surface of the vapor chamber is rectangle, theoretically the center should have the highest temperature while the corners have almost the lowest. Thus, for both the bottom and top surface, the temperatures at these five representative points are tested, which is similar with the work of Wang et al. [8]. Additionally, another two test points (T6-T7) on the top surface are used for further check of the temperature uniformity.

Test

In the experiments, the heat flux and the temperature distribution of vapor chamber were needed for evaluation. In order to obtain the heat flux of the vapor chamber, four thermocouples were inserted in the four holes of the copper rod with a depth of $0.5 \mathrm{~cm}$, achieving the centre, and a same distance from each other. As shown in Figure 4(a). Thus, four temperatures in the central axis of copper rod could be obtained.

As the thermal insulation prevented heat loss from radial direction, the heat transfer in the copper rod could be simplified to be one-dimensional situation. According to Fourier's Law, one-dimensional heat flux can be calculated as

$$
\Phi=-\lambda A \frac{\partial t}{\partial x}
$$

where $\lambda$ is the thermal conductivity of copper. $A$ is the sectional area of the copper rod. $\partial t / \partial x$ is the temperature gradient in the copper rod at stable situation. Figure 5 shows the linear fitting data of the present experiments. Based on the obtained temperature gradient from the method of linear fitting shown in Figure 5, and according to equation (1), the heat flux $\Phi$ can be obtained.

The temperature distribution of both bottom surface and top surface of the vapor chamber was also tested. Figures 4(b) and (c) show the test points on them. When all the temperatures were obtained, the thermal resistance including spreading thermal resistance and bulk thermal resistance could be calculated. Also, the temperature uniformity of condensing surface could be evaluated. The concrete principle was as follows.

The spreading thermal resistance and bulk thermal resistance are given by [5]

$$
R_{s}=\frac{t_{b \max }-\overline{t_{b}}}{\Phi}
$$




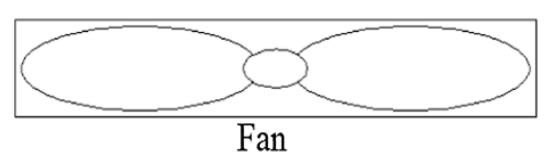

Data acquisition system

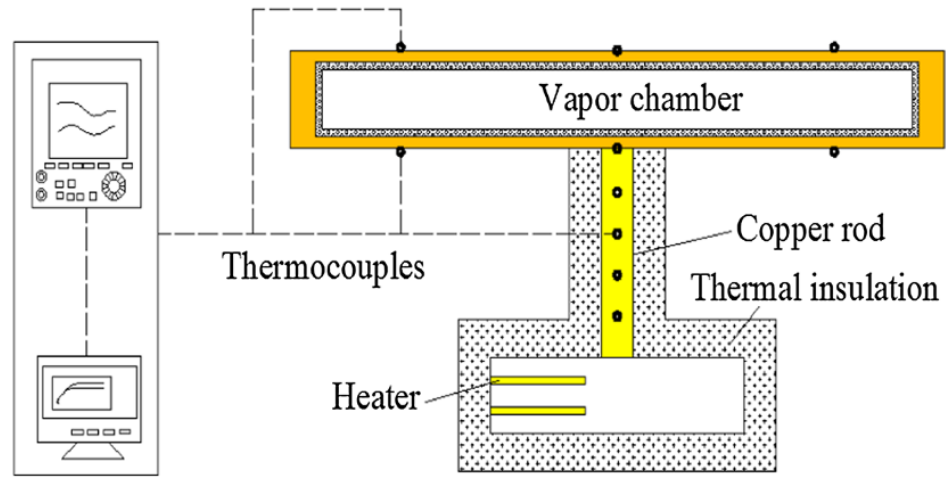

Figure 3 Schematic diagram of experimental system.

$$
R_{b}=\frac{\bar{t}_{b}-\bar{t}_{t}}{\Phi}
$$

where $R_{s}$ is the spreading thermal resistance, $R_{b}$ is the bulk thermal resistance, $t_{b \text { max }}$ is the maximum temperature on bottom surface, $\bar{t}_{b}, \bar{t}_{t}$ are the average temperature of the bottom and top surface respectively, $\Phi$ is the heat flux.

Thus, the total thermal resistance of vapor chamber $R_{V C}$ in this experiment could be calculated as below,

$$
R_{V C}=R_{s}+R_{b}
$$

After all the thermal resistances and temperature distribution on condensing surface under different inclination angles were obtained, the effect of inclination angle on the performance of the vapor chamber could be evaluated effectively.

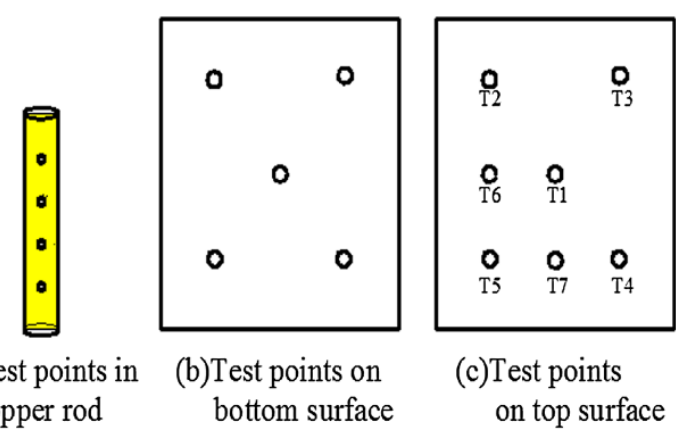

Figure 4 Temperature test points, (a) in copper rod, (b) on bottom surface of vapor chamber, (c) on top surface of vapor chamber. 


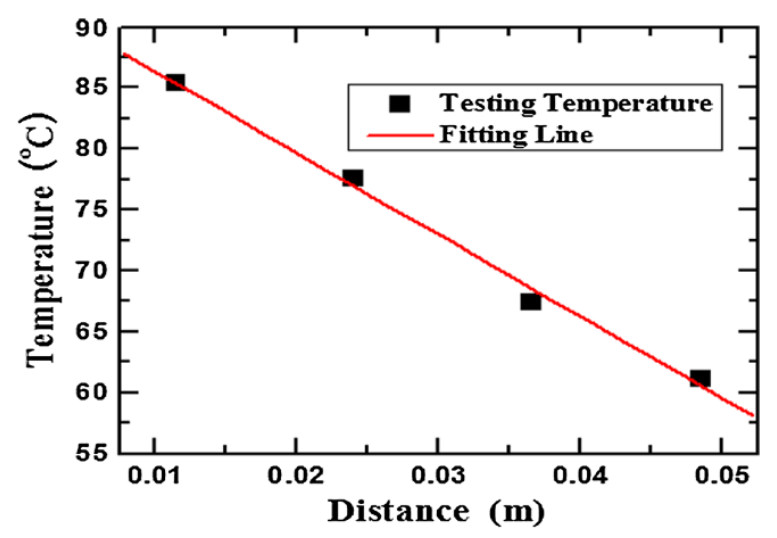

Figure 5 Linear fitting of the temperatures in the heating copper rod.

\section{Evaluation method}

To evaluate the effect of inclination angle on thermal performance of the vapor chamber, only the magnitudes of thermal resistance and temperature were not enough. The variation trend of them with the change of inclination angle was more important. Thus, the maximum relative change rate and the relative standard deviation were adopted to evaluate the degree of the change. They were given by

$$
\begin{aligned}
& R S D=\frac{\Delta m_{\max }}{\bar{m}} \\
& S D=\sqrt{\frac{\sum_{i=1}^{n}\left(m_{i}-\bar{m}\right)}{n-1}} \\
& R S D=\frac{S D}{\bar{m}}
\end{aligned}
$$

where, $R C R$ is the maximum relative change rate, $S D$ is the standard deviation, $R S D$ is the relative standard deviation, $m_{i}$ is the $i$ th value in a group of data, $\bar{m}$ is their mean value, $n$ is the total number of the data group, $\Delta m_{\max }$ is the maximum difference relative to average value.

\section{Accuracy analysis}

Based on the thermal resistance equation, the heat flux and temperature differences influence its calculation accuracy. In other words, the measurement accuracy of the heat flux and temperature is the key.

According to Figure 5, because of good thermal insulation, the accuracy of the linear fitting is about $99.72 \%$. This shows that the heat flux measurement has very high accuracy. In addition, according to the present evaluation method, we compared the thermal resistance and the temperature under different inclination angles, in all tests, the heating and thermal insulation are exactly the same. The variations of the heat fluxes under different inclination cases nearly do not exist. Therefore, the measurement error of the heat flux nearly can be ignored for making conclusions in this paper.

In respect to the measurement accuracy of temperature, the errors mainly lay in the measurement error of the thermocouples and the reading error of the digital data 


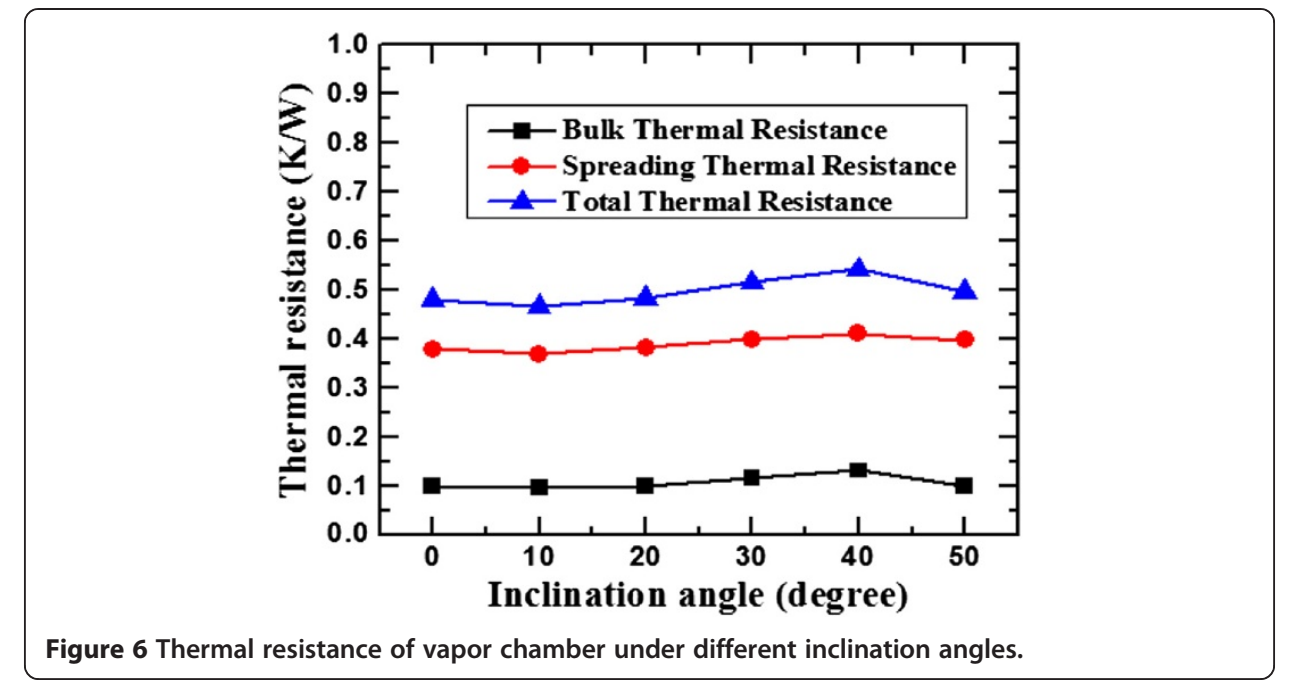

acquisition system. When the temperature was in the range from -30 to 150 , the measurement error of T-type thermocouple was about 0.2. The data acquisition system had a reading error of 1 since the cold junctions of the thermocouples used the default setup supplied by the system, and not the ice bath with constant 0 . Therefore, the total error of the temperature measurement was about $1.2^{\circ} \mathrm{C}$. According to the work of Huang et al. [7] and Wang et al. [8], which have almost the same test points, the maximum error for the thermal resistance is about $\pm 5 \%$.

\section{Results and discussion}

Figure 6 shows the thermal resistance of vapor chamber in different inclination angles. The $\mathrm{x}$-coordinate is the inclination angle, and the y-coordinate is the thermal resistance. As shown in Figure 6, both the spreading thermal resistance and bulk thermal resistance were considerably small, and the former was about four times as large as the latter. Thus, for the thin plate heat spreader, the decreasing of the spreading thermal resistance could have greater contribution to increase heat dissipation. From Figure 6, it is also found that as the inclination angle varied, both two kinds of thermal resistances fluctuated little. They were close to those without inclination. Because the total thermal resistance of the vapor chamber was the sum of spreading thermal resistance and bulk thermal resistance, the total thermal resistance was also given for overall assessment. In Figure 6, it could be seen that the total thermal resistance of the vapor chamber was only about $0.5(\mathrm{~K} / \mathrm{W})$. As the inclination angle varied, the total thermal resistance had little change as well. The maximum relative change rate is $9.1 \%$ while the relative standard deviation is $5.5 \%$, as listed in Table 2 .

Table 2 Thermal resistance and temperature uniformity under different inclination angles

\begin{tabular}{lll}
\hline Inclination angle $\left({ }^{\circ}\right)$ & $\boldsymbol{R}_{\boldsymbol{V C}}(\mathrm{K} / \mathrm{W})$ & SDof top surface temperature $\left({ }^{\circ} \mathrm{C}\right)$ \\
\hline$R C R(\%)$ & 9.1 & 4.5 \\
$R S D(\%)$ & 5.5 & 3.7 \\
\hline
\end{tabular}




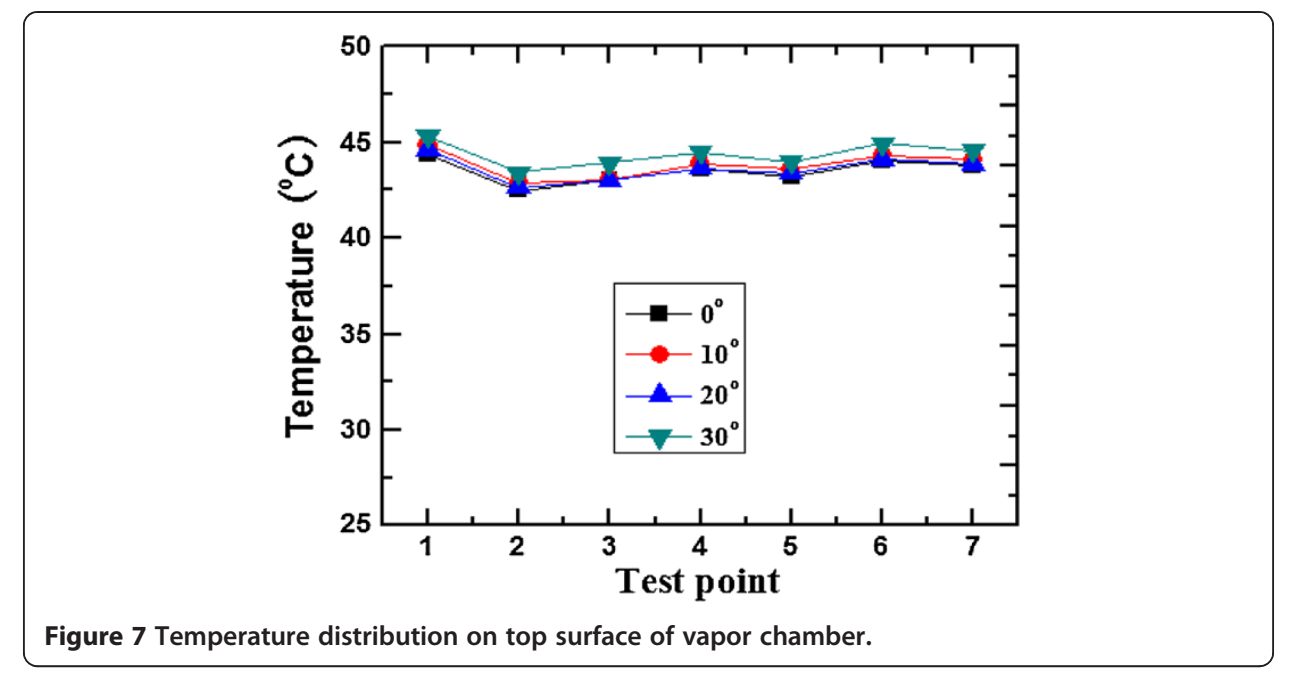

Figure 7 shows the absolute temperatures on the top surface of vapor chamber under different inclination angles. The $\mathrm{x}$-coordinate is the test point, and the $\mathrm{y}$-coordinate is temperature. Because all the data closely gathered together, just the temperatures under four inclination angles were plotted in Figure 7. As shown in Figure 7, the temperature at all test points for various angles were nearly the same, the largest difference was less than 2 . This confirmed that the vapor chamber could achieve good temperature uniformity on the condensing surface. It is also noted that the temperature of test point 1 (T1) was higher than that at other test points under four inclination angles. It can be explained as following. The test point 1 (T1) was located at the center of support copper column and heat source, as shown in Figure 4(c) and Figure 2. Thus, the heat could be transported to the point 1 more effectively compared to other test points. In Figure 7, we also noted that the temperature data at various test points under different inclination angles were close to each other, and this proved that the thermal performance of the vapor chamber was stable under different inclination angles. In order to have more clear analysis, for the effect of the inclination angle on the top surface temperature uniformity, which was evaluated with the standard deviation $(S D)$ of the seven tested temperatures, the maximum relative change rate and relative standard deviation were also calculated. As listed in Table 2, they are $4.5 \%$ and $3.7 \%$ respectively.

\section{Conclusions}

In this paper, experiments were conducted to analyze the effect of inclination angle on the performance of a kind of vapor chamber. The thermal resistance and temperature uniformity under different inclination angles were evaluated. The test results showed that the temperatures at all test points for various angles were very close, the largest difference was less than 2. It demonstrated that the present vapor chamber has ability to effectively decrease hotspot phenomena in high heat flux electronic packaging. 


\section{Acknowledgments}

The authors would like to express their great thanks to the support from 973 Project of The Ministry of Science and

Technology of China (2011CB013105) and in part by National Science Foundation of China (51376070).

Received: 3 February 2014 Accepted: 23 June 2014

Published online: 06 September 2014

\section{References}

1. Go JS: Quantitative thermal performance evaluation of a cost-effective vapor chamber heat sink containing a metal-etched microwick structure for advanced microprocessor cooling. Sens Actuators A 2005, 121:549-556.

2. Koito Y, Imura H, Mochizuki M, Saito Y, Torii S: Fundamental experiments and numerical analyses on heat transfer characteristcs of a vapor chamber (effect of heat source size). JSME Int J Series B 2006, 49(4):1233-1240.

3. Wu XP, Mochizuki M, Nguyen T, Satio Y, Wuttijumnong V, Ghisoiu H, Kumthonkittikul V, Sukkasaem P, Nimitkiateklai P, Kiyooka F: Low profile-high performance vapour chamber heat sinks for cooling high-density blade servers. In 23rd IEEE SEMI-THERM Symposium, IEEE; 2007:174-178.

4. Zhang M, Liu ZL, Ma GY: The experimental and numerical investigation of a grooved vapor chamber. Appl Therm Eng 2009, 29:422-430.

5. Huang HS, Chiang YC, Huang CK, Chen SL: Experimental investigation of vapor chamber module applied to high-power lighting-emitting diodes. Exp Heat Transf 2009, 22:26-38.

6. Wong SC, Huang SF, Hsieh KC: Performance tests on a novel vapour chamber. App/ Therm Eng 2011, 31:1757-1762.

7. Luo XB, Hu R, Guo TH, Zhu XL, Chen W, Mao ZM, Liu S: Low Thermal Resistance LED Light Source with Vapor Chamber Coupled Fin Heat Sink. In Electronic Components and Technology Conference. Las Vegas, NV, USA: ECTC60; 2010:1347-1352.

8. Wang RT, Wang JC, Chang TL: Experimental analysis for thermal performance of a vapour chamber applied to high-performance servers. J Mar Sci Technol 2011, 19:353-360.

doi:10.1186/s40539-014-0012-7

Cite this article as: Ma et al:: Effect of inclination angle on the performance of a kind of vapor chamber. Journal of Solid State Lighting 2014 1:12.

\section{Submit your manuscript to a SpringerOpen ${ }^{\circ}$ journal and benefit from:}

- Convenient online submission

- Rigorous peer review

- Immediate publication on acceptance

- Open access: articles freely available online

- High visibility within the field

- Retaining the copyright to your article

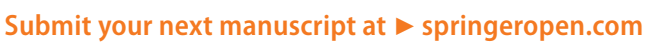

